\title{
15. L'ethos de crédibilité chez les candidats à la présidence : l'exemple du pronom je présidentiel dans le débat Hollande-Sarkozy 2012
}

Malin Roitman

Stockholms universitet

\section{Introduction}

\subsection{But et fondements de l'étude}

Le débat de l'entre-deux-tours reste un événement décisif pour les élections présidentielles. Cette étude vise à étudier comment les candidats aux présidentielles de 20I 2 renforcent leur crédibilité, leur ethos, dans l'interaction. Nous étudierons plus particulièrement l'emploi du pronom je tel qu'il est employé dans le débat par les candidats, un élément qui renvoie le plus explicitement à un sujet JE en chair et en os derrière le discours. La deuxième étape sera d'analyser la relation entre l'ethos et l'utilisation rhétorique de la négation de phrase ne...pas, le plus important et le plus fréquent outil de contre-argumentation dans

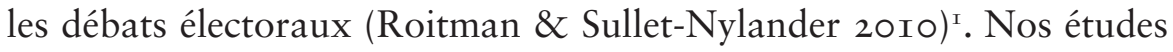
ont montré que la contre-argumentation par négation est un paramètre qui est pertinent pour la détermination du type de texte (polémique) et du genre (débat politique). L'étude vise donc à mettre l'accent sur la relation entre, d'une part, la construction de la crédibilité telle qu'elle est présentée à travers les je des candidats et, d'autre part, la réfutation des arguments de l'un et de l'autre candidat.

Les questions que nous nous sommes posées pour formuler l'objectif de l'étude sont :

- Quelles sont les formes utilisées pour exprimer l'ethos dans le débat?

Comment citer ce chapitre :

Roitman, Malin, L'ethos de crédibilité chez les candidats à la présidence : l'exemple du pronom je présidentiel dans le débat Hollande-Sarkozy 20I2. In: Engwall, Gunnel \& Fant, Lars (eds.) Festival Romanistica. Contribuciones lingüísticas - Contributions linguistiques - Contributi linguistici - Contribuições linguísticas. Stockholm Studies in Romance Languages. Stockholm: Stockholm University Press. 20I 5, pp. 29I-3 I6. DOI: http://dx.doi.org/Io.I6993/bac.o. License: CC-BY 
- Quelle est l'importance du genre « débat politique » pour l'établissement de l'ethos?

- Quel est le rôle que jouent les représentations préétablies des candidats pour leur ethos?

- Quelle est la relation entre la négation et l'ethos des candidats à la présidentielle?

L'objectif global est d'élargir les connaissances et d'approfondir la compréhension de la construction de l'ethos et de la contre-argumentation dans le discours politique français, et de relever les mécanismes linguistiques et rhétoriques qui y sont en jeu.

\subsection{L'ethos et je dans un débat politique, pour quoi faire?}

Un candidat présidentiel a besoin de paraître crédible. Pour convaincre les citoyens qu'il y a, derrière les beaux discours, une personnalité et un projet politique sérieux et pour lesquels on votera, c'est dans le discours qu'il faut se donner une image crédible. La construction d'un ethos rhétorique de crédibilité implique, à en croire les propos aristoteliens, les valeurs associées aux qualités personnelles et professionnelles comme la franchise, la droiture, la vertu, la confiance, le bon sens, l'humilité, la responsabilité, la force, etc. (Charaudeau \& Maingueneau 2002 : 238 ; Maingueneau I999 et 2002).

Ce n'est pas en effet très recherché d'étudier le pronom je quand on veut connaître les mécanismes de la présentation de soi des candidats présidentiels. Dans les élections présidentielles, il est évidemment question de choisir une seule personne et en arrivant au deuxième tour des élections, on a l'impression que les médias ne focalisent plus tellement sur les partis politiques mais sur les deux candidats qui restent ; il s'agit d'élire le chef de l'État, la fonction avec le plus de pouvoir officiel et le plus grand prestige dans la République française.

Nous avons trouvé particulièrement intéressant de continuer sur cette piste après avoir appris dans une étude effectuée par Véronis (20I2) que l'usage des je de Sarkozy a augmenté considérablement pendant les trois périodes de candidature dans sa carrière (la première pour l'UMP 2004, la deuxième et troisième pour la présidence, 2007 et $\left.20 \mathrm{I} 2^{2}\right)$. L'étude lexicométrique de Véronis est basée sur 728 discours politiques de Sarkozy entre les années 2004 à $20 \mathrm{I} 2$ (et de Hollande de janvier à mai de 20I2). Ses chiffres montrent que l'usage de je va croître de façon intense jusqu'au soir du second tour des élections présidentielles et aussi que les je déclinent ensuite de façon progressive (Véronis 
20I2). Le nombre des je de Sarkozy atteint le même niveau à la fin de la campagne de 2012 que pendant la même période dans la campagne de 2007.

Cela va de soi que le pronom personnel je fasse partie des formes en rapport avec la construction de l'ethos dans le duel présidentiel. Les je explicites du discours devraient ainsi introduire l'image que les candidats veulent se donner. L'emploi de $j e$ dans les discours présidentiels a également été le sujet d'études antérieures, en lexicométrie et en analyse de discours. Adam (I999 : I39-155) a étudié l'ethos dans les discours du président de Gaulle, dans ceux de Giscard d'Estaing et de Mitterrand, à la base d'un modèle qui prend en compte leurs usages de connecteurs argumentatifs, d'actes performatifs et de pronoms personnels. Fiala \& Leblanc (2004) et Leblanc (2005) ont fait des études en lexicométrie sur les verbes et les adjectifs qui émergent avec les je, nous et vous des discours présidentiels. Ils y ont trouvé une certaine ritualisation dans l'usage de certains je plutôt faibles de sens : "ce que je voudrais...». Marchand et Dupuy (2009, 20II, 20I2) et Véronis (2007, 20I2) ont également, dans une démarche lexicométrique, étudié l'usage des pronoms, entre autres unités, dans les débats de l'entredeux-tours. Nous ne trouvons cependant pas d'études approfondies, telle que la nôtre, sur le fonctionnement des pronoms, en analyse du discours.

\section{Cadre théorique}

\subsection{L'ethos - les possibilités et les frontières d'une notion}

Le terme ethos tel qu'il est employé en analyse du discours, renvoie à un concept qualitatif utilisé pour décrire toutes les formes d'expressions explicites avec lesquelles le sujet parlant établit la crédibilité de son message (Charaudeau \& Maingueneau 2002) ? $^{3}$ Dans cette étude, la notion de « ethos » sera limitée aux expressions linguistiques, vues dans leur contexte générique.

L'ethos est défini chez Aristote (I99I ${ }^{4}$ comme les éléments qui facilitent la confiance en celui qui parle, notamment : le bon sens, la vertu et la bienveillance. Il y a déjà chez Aristote l'idée d'une division entre un ethos pré-discursif et un ethos discursif. Le premier est constitué des images et des idées collectives, des lieux communs, des qualités associées à une position spécifique dans la société mais aussi à une personne. Le concept d'ethos pré-discursif, qui a été retravaillé en analyse du discours, est ainsi nourri des « connaissances » communes 
à une communauté linguistique, sociale et culturelle, plus ou moins identifiables (Maingueneau 2002 : 238 ; Amossy 2010)5. La crédibilité des candidats est fondée sur l'ethos pré-discursif ainsi que sur ce que nous appelons l'ethos discursif, à savoir la représentation effective de soi chez l'auditoire, influencée par les contextes génériques et sociaux de l'événement communicatif même. Cette dimension de l'ethos surgit ainsi dans le débat même, comme le résultat de l'usage d'un discours qui soit consolide, soit rectifie l'ethos pré-discursif ; il s'agit en d'autres termes de la matérialité linguistique, le discours effectif. Même si l'ethos dans un sens global touche aux questions de l'identité, il s'agit, dans l'analyse du discours, d'étudier l'ethos tel qu'il apparaît dans le discours, à savoir la présentation discursive que les candidats font d'eux-mêmes et de leur qualités - et non pas l'analyse de la psychologie et de l'éthique de la personne réelle derrière le texte. Il s'agira ici d'analyser le jeu de l'interaction entre l'ethos discursif et l'ethos pré-discursif.

Amossy (2010:42) dit que l'ethos est " une dimension intégrante du discours ", que toute prise de parole implique une "présentation de soi $»^{6}$. Elle parle aussi de l'ethos rhétorique (instrumental), à savoir l'efficacité d'un discours avec un but précis, comment ce que l'on dit et la manière dont on le dit seront efficaces pour l'objectif, dans une situation de discours où l'on veut convaincre, tel que dans le discours politique et publicitaire. Les enjeux de l'ethos discursif sont ainsi les suivants : le discours, dans le sens production orale ou écrite, sera toujours une présentation indirecte et souvent inconsciente de l'identité du locuteur. De l'autre côté, l'ethos peut être intentionnel, c'est-à-dire le résultat d'un travail où l'image du locuteur est élaborée pour que le texte soit convaincant. L'ethos représente ainsi un aspect du style rhétorique que doit prendre l'orateur pour capter l'attention et gagner la confiance de l'auditoire, pour se rendre crédible et sympathique. Il s'adresse à l'imagination de l'auditoire pour appeler à son pathos (Amossy 2010 : 72 ; 2000 : 86). Amossy (2010) fait noter, à l'instar de Maingueneau (I999, 2002), que l'ethos (pré-discursif et discursif) en analyse du discours concerne l'image discursive transmise d'une personne et non pas celle de la personne réelle.

Amossy (2010 : IO) insiste aussi sur l'investigation des discours en « je ", étant donné qu'elle " permet de voir comment l'identité se construit dans l'échange verbal, comment elle [l'identité] se négocie par rapport à l'autre, en quoi elle est liée à des questions d'efficacité discursive ». 


\subsection{Ethos rhétorique et le genre débat politique}

Nous soulignons, comme Adam (I999), Charaudeau (200I) et Maingueneau (I998) entre autres, l'importance d'inclure les paramètres des genres textuels pour toute description des phénomènes discursifs, $\mathrm{y}$ compris l'ethos. Amossy (20I0:9) est du même avis lorsqu'elle dit que «la présentation de soi » est " une construction discursive » : le locuteur se positionne et construit une identité dans le discours et avec le discours et il se positionne également dans un espace social et générique. Nous partirons de l'idée que le débat présidentiel serait conditionné par certaines contraintes discursives, culturelles et institutionnelles de ce genre particulier (Charaudeau 200I).

Maingueneau (I998 : 60-64) a élaboré un modèle où il met les phénomènes discursifs, comme l'ethos, en relation avec la scène d'énonciation d'un discours. Celle-ci implique I) "la scène englobante " qui représente le type de discours dont il s'agit, à savoir sa fonction visée - véhiculée par certains traits formels : discours narratif, pédagogique, argumentatif ; 2) "la scène générique » qui représente les normes sociales et culturelles concernant la forme textuelle, la distribution de rôles préétablis et à l'aide desquelles on libelle les textes : roman, manuel, essai, etc. ; finalement (3) la "scénographie " qui représente les formes de présentation des messages. Maingueneau propose que l'interprétation de l'ethos - ainsi que d'autres phénomènes rhétoricodiscursifs - soit analysée à la lumière des trois paramètres.

En appliquant ces concepts à nos matériaux, il faut comprendre qu'un texte argumentatif comme le débat politique (la scène englobante) vise à persuader et que les $j e$, qui renvoient au sujets empiriques, sont liés au positionnement (idéologique, argumentatif) du discours. Il faut aussi considérer que le débat électoral est un genre (la scène générique) interactif avec des tournures linguistiques souvent formalisées, voir ritualisées. L'interaction peut, en dernier lieu, être mise en scène de différentes façons (la scénographie), et les interlocuteurs peuvent prendre différents rôles selon le type de texte : père-fils, professeur-élève, savant-ignorant, etc. C'est à la lumière de ces trois scènes d'énonciation que nous essaierons d'étudier les dispositifs de l'ethos rhétorique de crédibilité chez les candidats. Maingueneau (I998) fait aussi noter qu'il faut voir les deux dimensions de l'ethos - discursif et pré-discursif - à la lumière du concept de genre.

\subsection{Ethos et éléments de langue}

La présentation de soi peut être faite de façon directe ou indirecte. Benveniste (I966:252) montre que le locuteur-sujet parlant laisse des 
traces dans son discours ; c'est à l'aide de certaines unités linguistiques, surtout les déictiques, que le sujet surgit dans le discours ; la subjectivité par excellence s'inscrit à travers le pronom personnel je. Il est cependant et probablement plus rare que l'on dise explicitement « je suis une personne honnête » que de le montrer dans les arguments, le ton, ou à travers des choix de mots, etc. Ce qui a fait couler beaucoup d'encre dans le domaine de l'énonciation est la double nature du pronom je (Barthes 1970 : 212 ; Ducrot 1984 : 199); il s'agit du fait que d'un côté, le locuteur se prend comme thème de son propre discours, le je est alors un être du discours, celui dont il est question. De l'autre côté, le locuteur se présente dans tout ce qu'il dit, dans l'intonation, l'attitude, les choix de mots, à savoir toute modalité qui renvoie au sujet empirique derrière le discours. Ducrot a fait observer que les expressions qui témoignent de l'ethos (attitudes, jugements de valeur, etc.) sont attribuées au locuteur, celui qui est responsable de l'énonciation et celui quiexplicite ou pas - renvoie au sujet parlant, le $J E$ empirique derrière le texte. Kerbrat-Orecchioni ( $1980: 32$ ) s'intéresse aux "subjectivèmes ", notamment les unités de langue qui dénotent le sujet parlant derrière le discours sans que celui-ci apparaisse explicitement ; ce sont les mots affectifs, axiologiques et évaluatifs. Constatons ainsi que l'ethos du locuteur-sujet parlant (dans le cas de débat c'est le même) s'exprime même s'il n'y a pas de je explicite.

Cette division, entre, d'un côté, locuteur d'énoncé et l'ethos dit et, de l'autre, locuteur d'énonciation et l'ethos montré, a été explorée dans les modèles de polyphonie sous différentes dénominations (Ducrot I984: 20 I Nølke et al. 2004 ; Maingueneau 2002). De cela suit la distinction faite entre ce que nous appellerons dorénavant je-dits et je-montrés. Le je-dit renvoie aux êtres du discours, les protagonistes de l'interaction et constitue celui qui présente les thèmes de l'événement communicatif. Le je-montré renvoie au sujet empirique et a une fonction métalinguistique ; il se positionne dans le discours, vis-à-vis des arguments présentés et du déroulement de l'interaction ; c'est le metteur en scène des énoncés qui s'impose explicitement, en commentant son propre discours et argumentation, ainsi que celui de l'autre. C'est une fonction assez importante dans les débats, où les candidats doivent se positionner, maîtriser, prendre et garder la parole. Voilà trois exemples de ces deux types à titre d'illustration :

Hollande : je dois dire [je-montré] ce soir quel président je serai [je-dit] si les Français m'accordent leur confiance. 
Hollande : J'en arrive [je-montré] au droit de vote après cette digression. Sur le droit de vote, c'est une position que je défends [je-dit] depuis des années.

Sarkozy : Je ne suis pas votre élève [je-dit]. J'y répondrai [je-montré] après vous avoir dit ce que j'ai à vous dire [je-montré].

Dans l'analyse nous tenterons de voir ces types de « je » explicites à travers les niveaux génériques proposés par Maingueneau (I998).

\subsection{Hypothèses}

La première hypothèse générale est comme suit : plus le décalage entre l'ethos pré-discursif et l'ethos effectif est grand, plus coûteuse sera la présentation de soi dans le discours. Plus les deux ethos se correspondent l'un à l'autre, moins coûteuse sera la présentation de soi. Sarkozy a un ethos pré-discursif assez chargé et négatif, et Hollande a, dans son rôle de débutant dans cette course, un ethos pré-discursif moins important et moins négatif. Ce dernier a conséquemment moins à défendre.

Étant donné que le débat est un genre interactif et argumentatif avec deux adversaires, il est assez naturel que le président sortant se retrouve dans une position de défense. Nous pensons ainsi que l'ethos des candidats n'est pas toujours un ethos rhétorique voulu mais qu'il résulte aussi d'une certaine "défense de soi » dans cette confrontation. Ainsi, la négation semble jouer un rôle important quant à l'ethos rhétorique des candidats.

\section{Analyse des deux faces du je et l'ethos rhétorique}

Dans l'objectif de se présenter comme un futur président qui accomplira ses devoirs - qui transformera ses promesses en action, qui écoutera et essaiera de répondre aux exigences des citoyens, et qui aura la force de réaliser ses projets - les stratégies sont nombreuses. Nous avons donc choisi d'analyser un élément de langue primordial pour l'ethos, notamment le pronom de la première personne au singulier, le je. Nous opérons avec les deux catégories de je explicites : les je-dits représentent les deux protagonistes et forment, au niveau de l'énoncé, avec leurs verbes les thèmes du discours. Ainsi, on peut les contredire, être d'accord, etc. Les je-montrés relèvent de l'énonciation - dans le sens de Ducrot et explicitent le sujet parlant physique derrière le discours. Ces deux types de je témoignant de l'ethos sont toujours analysés en prenant en considération les verbes accompagnant. D’après la sémantique et la valeur argumentative des verbes accompagnants, nous avons catégorisé 
et quantifié les je des deux candidats. Le Tableau I montre ainsi la répartition des je-ethos du corpus examiné.

Tableau 1. La répartition des ethos rhétoriques des je

\begin{tabular}{lrrr}
\hline Les je-dits : & Hollande & Sarkozy & Total \\
\hline$J e$ et l'ethos du rassemblement & $\mathrm{I} 8$ & 3 & $2 \mathrm{I}$ \\
$J e$ et l'ethos du leadeur respectueux et humble & $3 \mathrm{I}$ & $\mathrm{I} 8$ & 49 \\
$J e$ et l'ethos de la sincérité-fidélité & 9 & 4 & $\mathrm{I} 3$ \\
$J e$ et l'ethos de la politesse & 9 & 5 & $\mathrm{I} 4$ \\
$J e$ et l'ethos de l'autorité & 8 & $\mathrm{I} 5$ & 23 \\
$J e$ et l'ethos de l'engagement émotionnel & 3 & 3 & 6 \\
$J e$ et l'ethos de la volonté politique & 22 & $\mathrm{I} 4$ & 36 \\
$J e$ et l'ethos de l'idéologue & 46 & 37 & 83 \\
$J e$ et l'ethos de l'action et de la force & 56 & 45 & $\mathrm{IOI}$ \\
$J e$ et l'ethos de la responsabilité & 5 & 2 & 7 \\
$J e$ et l'ethos de l'indignation & 3 & $\mathrm{I}$ & 4 \\
Total je-dits & $2 \mathrm{II}$ & $\mathrm{I} 46$ & 357 \\
\hline Les je-montrés : & & & \\
\hline$J e$ et l'ethos de la maitrise du discours & 28 & $\mathrm{I} 8$ & 46 \\
$J e$ et l'ethos du positionnement du pouvoir & 39 & 45 & 84 \\
Total je-montrés & 67 & 63 & $\mathrm{I} 30$ \\
Total je-montrés et je-dits & & & 487 \\
\hline
\end{tabular}

Dans le Tableau I, nous avons catégorisé les différents je-dits à partir des prédicats qui les qualifient et avec lesquels ils expriment un ethos de crédibilité. En voici deux exemples de la catégorisation : le propos « Je préfère tenir bon sur une position que je défends depuis des années » (Hollande) exprime l'ethos de la sincérité-fidélité ; le propos " Hier, je me suis adressé à tous les Français » (Sarkozy) exprime l'ethos du rassemblement. Ces « je » présentent les candidats, donnent l'image discursive de leur personnalité, de leurs opinions, de leurs projets politiques et de leurs capacités à les réaliser. Les je-montrés sont en revanche une catégorie plus fonctionnelle; ils positionnent les sujets parlants dans l'événement de communication : "Je vais vous dire une chose, monsieur Hollande : c'est une fierté... » (Sarkozy), "Est-ce que je dois vous rappeler que vous êtes au pouvoir depuis dix ans »(Hollande). 
En reprenant le schéma sur la scène d'énonciation tripartite de Maingueneau, nous sommes partie de l'hypothèse que les phénomènes discursifs sont conditionnés par le contexte socio-culturel, par le genre (les formes linguistico discursives conventionnelles au débat politique) et les formes de présentation choisies. Pour l'analyse des ethos nous prenons ainsi en considération le cadre institutionnel de la fonction présidentielle et celui des élections (type de discours - scène globale), les thèmes évoqués, le vocabulaire, procédés énonciatifs (scène générique) et les types d'interactions (les scénarios choisis).

Analysons d'abord la répartition. Constatons qu'il y a quatre catégories qui dominent et d'autres catégories qui sont assez réduites mais qui apparaissent chez les deux candidats. Il ressort clairement que Hollande produit plus de je que Sarkozy dans la catégorie jedits. Hollande domine ou joue match nul dans tous les domaines sauf dans celui de "l'ethos de l'autorité ». Il semble avoir davantage besoin de se présenter devant les spectateurs, et il s'efforce plus pour faire passer ses visions politiques. Il nous semble aussi logique que c'est surtout le président sortant, Sarkozy, qui se présente comme l'autorité. Même si nous n'avons pas quantifié les je + négation séparément, nos comparaisons montrent que c'est surtout Sarkozy qui se sert de cette collocation, souvent pour se défendre. Ainsi, de façon préliminaire, notre première hypothèse se voit confirmée. Dans la catégorie je-montrés, nous constatons que la répartition est presque égale même si c'est Sarkozy qui emploie le plus les je-montrés du positionnement du pouvoir. Son rôle institutionnel de président de la $\mathrm{V}^{\mathrm{e}}$ République l'oblige de démontrer devant son peuple sa position et son pouvoir. Il nous semble ainsi naturel que Sarkozy présente un ethos d'un leader puissant, ayant été au pouvoir depuis cinq ans.

\subsection{Les je-dits}

Les domaines dominants des je-dits sont les je-action et force ( $\mathrm{I}-2)$, les je-idéologue (3-4) et les je-leadeur humble ${ }^{7}(5-6)$. Les ethos rhétoriques des candidats concernent ainsi avant tout l'image d'être l'homme d'action, d'avoir la force de réaliser les projets envisagés, de persister dans cette position, d'avoir des visions politiques pour le futur et en même temps d'avoir un caractère humble, à savoir la capacité d'écouter et d'avoir un dialogue avec son peuple et de les respecter, mais aussi de pouvoir admettre ses propres limites en face de problèmes sérieux : 
(I) Hollande : j'instaurerai un forfait de base qui fera que les consommateurs, jusqu'à un certain niveau, un volume de leurs consommations, paieront le même tarif.

(2) Sarkozy : j'ai pris un certain nombre d'engagements, les effectifs de la fonction publique seront stabilisés globalement tout au long du prochain quinquennat et la dépense publique n’augmentera pas de plus de I \% par an.

(3) Hollande : Je considère que 300 euros, sur un couple de smicards, c'est inacceptable.

(4) Sarkozy : ... j'estime qu'on ne réduit pas nos dépenses, nos déficits et nos dettes en envoyant en Europe des dettes qui seront garanties par qui, si ce n'est par les deux pays les plus forts de l'Europe, l'Allemagne et la France? C'est irresponsable.

(5) Hollande : J'ai une grande confiance pour l'industrie nucléaire, mais qui doit être portée sur les réacteurs de nouvelle génération.

(6) Sarkozy : Je veux leur parler en leur disant : vous avez exprimé un choix, ce n'est pas le mien, je vous respecte, je vous considère. J'ai entendu votre demande de nation, de frontières, d'authenticité, d'autorité, de fermeté.

L'ethos dont les candidats font le plus preuve est associé à l'action et à la force $(\mathrm{I}-2)$. En supposant que les candidats sont conscients de l'image qu'ils veulent faire passer dans le débat, ces qualités seraient ainsi celles qui donneraient le plus de crédibilité.

À la deuxième place viennent les je-idéologue; cet ethos est souvent négocié, à savoir que le fait de se présenter comme ayant des visions politiques est questionné par l'autre. L'ethos je-humble-quand les candidats se présentent comme proches du peuple, respectueux, et admettent la difficulté de la tâche de gouverner un pays - est aussi souvent négocié ; aussi bien Sarkozy que Hollande veulent s’approprier cet ethos mais chacun est contesté par l'autre. L'analyse discursive problématisent ainsi les quantifications du Tableau I ci-dessus : l'ethos effectif dépend notamment aussi des dispositifs de l'interaction, non seulement ceux de l'énoncé isolé. Voici un exemple d'une négociation de cet ethos :

(7) Sarkozy : Je pense enfin que ce débat doit être ce moment de démocratie où les Français en refermant leur télévision se disent : "On aime l'un, on préfère l'autre, on peut être en désaccord avec ce qu'ils 
disent mais ils ne nous ont pas menti, ils n'ont pas joué l'esquive, ils ont été vrais ». C'est ça notre défi, monsieur Hollande, être vrais.

Hollande : Oui, chacun aura sa vérité et chacun sera authentique, je n'imagine pas que vous feindrez.

Les catégories les moins importantes sont toutefois intéressantes, du moins dans un aspect comparatif. La politique n'est rien sans la volonté des femmes et hommes politiques de changer les domaines de la société qui marchent moins bien : les mauvaises finances, les injustices, les inégalités, etc. Dans le débat, les exemples de je-volonté sont assez nombreux alors qu'ils sont moins importants que les catégories ci-dessus :

(8) Hollande : je veux être le président qui redressera la production, l'emploi, la croissance.

(9) Sarkozy : Je veux mobiliser l'argent public, pas pour ceux qui ont déjà un emploi, pour ceux qui en ont besoin d'un.

(ıо) Hollande : je veux être aussi le président du rassemblement [...] je veux les réunir, car je considère que c'est de toutes les forces de la France dont nous avons besoin.

Un thème qui surgit et qui a été chaud pendant la campagne est celui du rassemblement (du peuple, des citoyens, des Français), ce qui a résulté en un ethos de je-rassemblement. Depuis l'arrivée de Sarkozy au pouvoir, on lui a attribué un ethos pré-discursif d'être le président qui, au contraire, a divisé le peuple français. C'est aussi sur cet ethos que joue Hollande dans son argumentation ; l'ethos de rassemblement est en effet le thème qui a été le plus négocié dans ce débat. Voilà deux extraits d'une plus longue séquence sur ce thème :

(I I) Sarkozy : Juste un mot sur le rassemblement. Le rassemblement, c'est un très beau mot, une très belle idée, mais il faut y mettre des faits. Le rassemblement c'est quand on parle au peuple de France, à tous les Français. Je ne suis pas l'homme d'un parti, je ne parle pas à la gauche. Hier, je me suis adressé à tous les Français.

(I2) Hollande : [...] je veux revenir sur le rassemblement, parce que je pense, comme vous, que c'est une notion essentielle pour notre pays. Et si vous avez le sentiment que pendant 5 ans vous avez rassemblé tous les Français, vous ne les avez pas divisés, vous ne les avez pas opposés, vous n'avez pas montré celui-ci du doigt, celle-là de certaine distance, alors je voudrais donner quitus. Mais je sais que les Français 
ont eu ce sentiment, d'avoir toujours à être soumis à des séparations, à des clivages.

Le fait qu'une interaction comme le débat est un événement de communication où l'ethos est négocié surgit très clairement ici. Chacun réclame l'ethos de rassemblement qui semble, dans ces élections, avoir une importante valeur symbolique, sans doute à cause de la lourde critique que Sarkozy s'est attirée pendant le quinquennat. Un composant dominant de l'ethos pré-discursif de Sarkozy, tel qu'il circule dans les médias, est justement son style présidentiel de " diviser pour mieux régner ", devenu un 'stigma discursif' dont il veut se débarrasser. Avec l'exemple ci-dessus, nous nous approchons inévitablement du rôle de la négation dans l'établissement de l'ethos, le thème du chapitre suivant. Celui-ci joue justement le rôle de négociateur ici ; aux spectateurs de juger qui sera celui qui rassemblera les Français.

Considérant la scène globale, constatons que les ethos des je-dits répondent à la fonction globale des interventions des candidats, notamment de montrer à l'électorat le vouloir, la force et les idées politiques qui pourraient changer les choses. Au niveau générique, constatons qu'il s'agit d'une certaine ritualisation comme les mêmes verbes reviennent : " je m’engagerai à », " Je serai un président.. », etc. En ce qui concerne la scénographie, il y a un jeu du pouvoir qui ressemble à un va-et-vient entre une fausse soumission ( $\mathrm{I}_{3}$ ) - de l'ironie - et le style autoritaire du leadeur omniscient et sarcastique (I4) :

(I3) Hollande : Donc, vous êtes très mécontent de vous. J'ai dû me tromper, j'ai dû faire une erreur. Je me mets à présenter mes excuses, vous êtes très mécontent de vous.

(I4) Sarkozy : Monsieur Hollande, je sais que vous avez le sens de l'humour mais vous, tenir bon sur vos convictions, franchement, pas vous, et pas ça.

Sarkozy domine ce jeu mais les deux candidats font preuve du sarcasme et de l'ironie.

\subsection{Les je-montrés et l'art de gouverner son discours}

Les je explicites de l'ethos montré opèrent la gestion de l'activité discursive et le positionnement du sujet parlant ; c'est un je méta-discursif qui commente son propre discours ainsi que celui du protagoniste. C'est le shifter par excellence, qui renvoie à la présence physique et psychique d'un sujet parlant JE, comme dans : " je dois dire ce soir 
quel président je serai » (Hollande). Dans le débat, nous rencontrons des formules comme "Je pose une question ", "Je viens sur votre proposition ", "Ce que j'attends du débat », "Je vais développer mon raisonnement " (Sarkozy) et "Mes propositions... j’y arrive ", " Je m'explique ", "Je vais venir maintenant sur la question » (Hollande). Ces je-montrés sont typiques pour les interactions publiques, où il s'agit de tenir un discours explicatif-pédagogique devant un auditoire, un discours qui risque d'être interrompu par l'adversaire. Avec ces $j e$, les candidats conduisent chacun leur propre discours - ils se positionnent et prennent de la place - en nous donnant des informations sur leurs activités discursives.

La plupart des je-montrés opèrent cependant un positionnement plus orienté vers l'argumentation, ils fonctionnent comme preuves de l'autorité derrière le discours du sujet parlant qui réclame avoir raison. Ces " je » renforcent les affirmations qu'ils introduisent et fonctionnent comme les expressions et verbes factifs, sous-entendant la "vérité » des arguments suivants :

(15) Hollande : Je rappelle que sous votre direction pendant 5 ans, la dépense publique a augmenté de $2 \%$.

(I6) Hollande : Je vous fais d'ailleurs observer qu'il y a des Français qui sont de culte musulman aujourd'hui.

(I7) Sarkozy : je vais vous dire pourquoi. Je vous l'ai expliqué.

(I8) Hollande : je vous dis qu'il vaut mieux une position intelligente qu'une position dogmatique.

(19) Sarkozy : Je vous dirai simplement trois exemples pour vous montrer que l'esprit du rassemblement irrigue toute la société française.

(20) Sarkozy : Je vous rappelle que ces talibans avaient décidé que les petites filles n'iraient plus à l'école.

Les je-montrés du débat exposent ici (2 $\mathrm{I}-23)$ une discussion métalinguistique concernant la répartition de la parole, non sans un ton ironique ; c'est ainsi qu'on reconnaît un débat :

(2I) Hollande : Je vous laisse terminer puisque vous voulez aller plus loin. Allez-y.

(22) Hollande : Je peux vous arrêter là-dessus ? Je peux vous arrêter làdessus ? Cette règle existe déjà pour les résidents communautaires. 
(23) Sarkozy : Si je peux terminer mon raisonnement...

Hollande : Répondez à ma question.

Sarkozy : Merci. Je vais terminer mon raisonnement.

Le je-montré est aussi un élément constitutif de la démonstration $d u$ pouvoir dans les débats. Il s'agit d'essayer de diriger l'interaction, d'être celui qui domine le discours. Ces formes de « je » transmettent l'ethos d'une personne qui montre la direction de son discours, qui se positionne, et qui par conséquent se donne l'image de pouvoir conduire son pays. L'émetteur des je-montrés est surtout Sarkozy :

(24) Sarkozy : Monsieur Hollande, vous avez parlé, sans doute pour être désagréable à mon endroit, d'un président normal. Je vais vous dire, la fonction d'un président de la République ce n’est pas une fonction normale.

(25) Sarkozy : Je vais vous dire une chose, monsieur Hollande : c'est une fierté. Il y a ceux qui parlent de rassemblement, et il y a ceux qui l'ont fait vivre.

Nous avons vu que ces je opèrent au niveau de la scène englobante, qui est liée à la fonction du texte - la visée persuasive. Ils sont aussi intéressants à étudier au niveau du genre textuel, comme des traits stéréotypés (de dominance, d'autorité : Je vais vous dire) dans les débats entre autorités dans un cadre politique. Exposons maintenant comment, de même qu'avec les je dits, les je montrés révèlent au niveau de la scénographie l'ambition d'instaurer des relations de pouvoir. Ainsi, nous voyons des attitudes ironiques, sarcastiques et d'autres stratégies de domination dans les propos introduits par ces je. Dans (26), sous un manteau ironique, Hollande met en scène une (fausse) soumission :

(26) Hollande : Donc, vous êtes très mécontent de vous. J'ai dû me tromper, j'ai dû faire une erreur. Je me mets à présenter mes excuses, vous êtes très mécontent de vous.

\subsection{L'ethos rhétorique négocié par négation : 'Je ne suis pas comme vous, je fais différemment'}

En nous appuyant sur Kerbrat-Orecchioni (2005 : 94), nous constatons que dans une interaction comme celle d'un débat politique, la construction de l'ethos, tel qu'il est conçu par les spectateurs, est à un certain degré une négociation, à savoir que les protagonistes brodent une image 
de soi, une image qui risque toujours de subir des contre-attaques. Amossy dit également que les protagonistes d'une interaction peuvent chacun « mettre [l'ethos] en doute, le corriger, le rejeter ou encore renvoyer une image alternative, éventuellement défavorable " (2010: I32). C'est à ce stade que la négation devient intéressante dans le discours des candidats. Nous allons voir comment la bipartition ethos pré-discursif et ethos discursif nous sera utile dans cette partie de l'analyse.

Avant d'expliquer le lien entre ethos et négation, il convient de présenter le postulat de base de la négation comme un opérateur polyphonique (Ducrot I984; Nølke et al. 2004). Selon cette vue, la négation stratifie son énoncé en deux points de vue superposés et hiérarchiquement organisés : l'un réfutant, l'autre réfuté. Voici une illustration de la polyphonie de la négation:

(27) Hollande : Non. Est-ce qu'il est du PPE ou non ? Répondez à ma question.

Sarkozy : Je ne suis pas votre élève.

La stratification de l'énoncé nié :

Point de vue I : 'Je suis votre élève'

Point de vue 2: 'Je ne suis pas votre élève'

Le point de vue I, l'affirmation du fait que 'je suis votre élève', est ainsi sous-jacent à l'énoncé nié. On conçoit cette affirmation comme une " voix ", ou point de vue, implicite à la négation, un point de vue qui peut être associé à un autre protagoniste que celui qui produit l'énoncé nié, dans ce cas à Sarkozy. Par un procédé ironique, Sarkozy, en faisant référence à la question précédente de Hollande, attribue le point de vue I à son adversaire, un point de vue que Sarkozy récuse. Le procédé peut se paraphraser ainsi : 'vous pensez que je suis votre élève mais je ne le suis pas'.

Si nous revenons à nos hypothèses, l'ethos rhétorique semble, en partie, être construit sur une contre-image défavorable qui est créée par la négation du candidat adversaire. Cette image se base souvent sur un ethos pré-discursif. La fonction rhétorique de la négation, reposant sur son statut de marqueur de polyphonie linguistique, semble ainsi pertinente pour l'examen de l'ethos des candidats ${ }^{8}$. Nous constatons que les exemples, où les candidats font allusion aux ethos pré-discursifs de l'autre ou d'euxmêmes, sont surtout limités aux je-rassemblement et aux je-leader humble mais touchent aussi quelques autres types d'ethos du débat. 
Ci-dessous, Hollande se donne l'image d'un président ouvert, réconciliant et coopérant, en qualifiant Sarkozy indirectement comme celui qui désunit les assemblées qui gouvernent, les différentes catégories professionnelles, les salariés, etc. Dans (28-29) il est fait référence directement à certains événements qui ont eu lieu. L'ethos pré-discursif du leadeur qui désunit le pays est nourri de la description dans les médias de Sarkozy et de son caractère péremptoire, un thème qui a aussi été récurrent dans la campagne électorale :

(28) Hollande : Moi je ne distingue pas le vrai travail du faux, les syndicalistes qui me plaisent et les syndicalistes qui ne me plaisent pas, les organisations patronales que je veux préférer ou d'autres. Non. Je n’oppose pas les salariés du privé ou du public.

(29) Hollande : Moi président de la République, je ne traiterai pas mon Premier ministre de collaborateur.

Ces exemples montrent que la négation permet de jouer sur l'ethos pré-discursif de l'autre candidat. Ainsi, l'ethos positif de Hollande, sous-jacent dans l'énoncé nié, est renforcé par l'image dévalorisante de Sarkozy qu'il fait passer, en se servant de la négation. C'est la mise en scène de cet ethos pré-discursif, véhiculé par le point de vue sous-jacent, et la réfutation du même qui donne de la force à ces exemples d'ethos rhétoriques. Constatons que la négation peut être un outil très économique pour la présentation de soi, étant donné qu'avec elle, le candidat fait d'une pierre deux coups : il se valorise en même temps qu'il dévalorise l'autre. Cette stratégie de la négation est d'autant plus efficace dans un débat où il est difficile pour l'adversaire de les contester. Il y aura ainsi un conflit entre l'image que Sarkozy, dans ce cas, élabore pendant le débat et l'image mise en scène par Hollande. C'est pourquoi nous dirons que la négation négocie l'ethos dans le débat. Étant donné que (28) est une façon indirecte de dire que Sarkozy sépare le vrai travail du faux, il est moins facile d'essayer de les rectifier que dans le cas où l'autre dit ouvertement que Sarkozy aurait tort de séparer le vrai travail du faut, etc. La négation, en qualité de phénomène de polyphonie, met en scène un propos sous-jacent, un ethos pré-discursif, qui peut être associé à ce stade à une voix collective adhérant à une certaine image de Sarkozy. Ce n'est donc pas seulement une idée inventée sur le moment par Hollande ; celui-ci joue justement sur ces ethos pré-discursifs et négatifs existants de Sarkozy, en disant que lui, Hollande, par contre, fera autrement. 
Voici deux exemples du même genre où Sarkozy se présente comme le candidat qui comprend et écoute les aspirations du "peuple ", et qui parle à tous les Français, sans exception :

(30) Sarkozy : Je ne suis pas l'homme d'un parti, je ne parle pas à la gauche. Hier, je me suis adressé à tous les Français, pas simplement aux syndicalistes qui ont parfaitement un rôle à jouer, le rassemblement c'est de parler à tous, y compris à ceux qui n'ont pas vos idées.

(3I) Sarkozy : Je n'ai pas pris une pince à linge pour me boucher le nez Monsieur Hollande. Je n'ai pas fait de leçon de morale à des gens qui vivent dans des quartiers où je ne vis pas, ou qui mettent leurs enfants dans des écoles où je ne vis pas. Pour moi, il n’y a pas des sous-citoyens.

En (30), Sarkozy joue sur un ethos pré-discursif selon lequel 'Hollande est l'homme d'un parti', ou 'Hollande parle uniquement aux gens de gauche', à la différence de Sarkozy qui est le président depuis un quinquennat et assume déjà en qualité de chef de l'État le rôle de celui qui rassemble le peuple. La négation fait d'une pierre deux coups : elle fait passer le message que Hollande reste un leader de gauche et que Sarkozy lui-même, est le leader de tous les Français. Sarkozy sous-entend, en se servant de la négation, l'ethos pré-discursif dévalorisant selon lequel 'Hollande vit loin (dans tous les sens) du peuple', 'il est snob et moraliste' ou bien 'Hollande méconnait la situation des gens désintégrés'. Au fond de ces deux exemples (3I-32), il y a les deux ethos pré-discursifs selon lesquels 'la gauche est un parti fermé' et aussi que 'la gauche sont des snobs intellectuels qui pensent toujours avoir la bonne réponse', auxquels Sarkozy a fait allusion à d'autres moments, entre autre dans le débat contre Ségolène Royal.

\subsection{L'ethos négocié : 'Je ne suis pas ce que vous prétendez que je suis'}

L'autre exemple du lien entre la négation polyphonique et l'ethos rhétorique est lorsque les candidats veulent se débarrasser d'un ethos pré-discursif défavorable attaché à leur propre personne. Il peut s'agir d'un ethos pré-discursif dans le sens d'image collective ou bien d'un ethos créé au cours du débat. La négation permet d'essayer de réfuter l'ethos pré-discursif, dévalorisant, attaché au locuteur même, et ainsi de corriger - négocier - son ethos :

(32) Hollande : Vous nous dites : "J'ai assumé un certain nombre de réformes difficiles ", mais à quel prix pour les Français ? À quel prix 
pour les injustices qui ont été creusées, les inégalités qui ont été aggravées ? [...] si un quinquennat se termine alors qu'il a été heurté et s'il n'y a pas eu comme vous dites de violences, je pense que le mérite en revient à toute la société française.

Sarkozy : Je n'ai pas évidemment le seul mérite, mais je ne peux pas être le seul coupable non plus Monsieur Hollande.

La négation introduisant l'énoncé de Sarkozy constitue une litote : c'est comme s'il adoucissait un ethos pré-discursif positif selon lequel 'Sarkozy a le seul mérite' et se présente ainsi comme le 'leadeur humble' qui ne prend pas tout le mérite à son compte. Dans le deuxième cas, il essaie de réfuter l'ethos pré-discursif qui lui est attribué par Hollande et d'autres, selon lequel 'il est le seul coupable'. Sarkozy négocie ainsi son ethos en jouant sur deux superlatifs : " le seul mérite » et " le seul coupable », ce qui renforce l'effet, d'un côté, d'une certaine omnipotence déguisée et, de l'autre, d'une victimisation dont se sert fréquemment Sarkozy.

La discussion sur la nécessité de rassembler un peuple divisé continue. L'ethos pré-discursif attaché au président sortant est que 'Sarkozy est responsable des injustices, des inégalités' ; c'est cette image que Hollande évoque dans sa réplique et dont Sarkozy essaie de se débarrasser en la réfutant. Ainsi les ethos pré-discursifs dévalorisants sont ici évoqués pour nuire à l'adversaire, et ces ethos sont ensuite négociés par celui-ci, à l'aide de la négation :

(33) Sarkozy : j'ai une preuve à vous donner de cet esprit de rassemblement qui m'a animé pendant 5 ans, c'est qu'il n'y a jamais eu de violence pendant les 5 ans de mon quinquennat, que je n'ai jamais été amené à retirer un texte qui aurait blessé ou créé un climat de guerre civile dans notre pays.

Sarkozy revient plus tard sur le même ethos pré-discursif qui lui est attaché et qu'il veut à tout prix faire disparaître, et il essaie de le faire en se servant de la négation :

(34) Sarkozy : Il y a eu des manifestations, elles se sont passées dans le calme, personne ne s'est senti humilié, il n'y a pas eu de divisions, il n'y a pas eu d'émeutes, il n'y a pas eu de violence.

Hollande n'a pas d'ethos pré-discursif aussi chargé que Sarkozy, ce qui donne une tâche plus difficile pour celui qui veut l'attaquer sur son passé. Sarkozy essaie cependant ici d'évoquer les liens d'amitiés entre Hollande et son prédécesseur à la candidature, Dominique 
Strauss Kahn, circonscrit par le scandale de New York, pour le rendre suspect :

(35) Hollande : Ce n'est pas moi qui ai nommé Dominique Strauss Kahn à la tête du FMI.

Sarkozy : Je le connais moins bien que vous.

Hollande : Mais je ne le connaissais pas plus [...] Vous pensez que vous connaissez la vie privée de vos collaborateurs ou de vos amis ? Moi pas. Je ne la connais pas.

Même si on peut penser que c'est un coup vulgaire de la part de Sarkozy, d'évoquer que Hollande a quelque chose à voir avec les agissements de DSK, il n'est pas sans risque pour Hollande d'avoir à se défendre contre cette allusion 'pas de fumée sans feu' évoquée par Sarkozy, de contredire un ethos pré-discursif plutôt innocent selon lequel 'Hollande connait DSK'. Étant données toutes les connotations qu'évoque l'ex-candidat et l'extension du mot connaître, cette (dé) négation "je ne le connaissais pas " serait ouverte aux interprétations qui peuvent amener assez loin, du type : 'je ne suis pas un perverti'. Les spectateurs se demandent éventuellement d'où vient cette nécessité de dénoncer les liens supposés avec DSK.

Nous avons donc évité de dire que ces ethos sont réfutés par la négation parce que, finalement, l'ethos rhétorique est un phénomène discursif qui appartient à ceux qui écoutent et évaluent le débat. Les débatteurs réfutent, déclarent faux - dans leur " univers de croyance »- les dits de l'autre mais, aux yeux des spectateurs, il faut considérer la négation d'un ethos du débat comme un procédé qui négocie l'ethos rhétorique des deux candidats. Nous sommes en face de deux candidats aspirant au poste de président et l'image qu'ils transmettent à l'audience est très importante. La scène du débat est celle d'un duel où les répliques sont des coups d'épée qui révèlent au public le style de la lutte, la force, la rapidité, la tactique des combattants, mais où le dernier coup mortel est donné par l'électorat.

\section{Discussions et conclusions}

4.1. Présentation de soi et je-ethos de crédibilité et les jeux du pouvoir L'ethos - la présentation de soi - est partout dans le discours ; lorsqu'un sujet parlant énonce, il ou elle énonce quelque chose sur soi. En regardant l'ethos à partir d'une forme spécifique de langue, comme nous l'avons fait avec je, nous avons éventuellement des réponses différentes 
dans le cas où nous aurions entamé une analyse d'une autre unité linguistique. L'objectif a cependant été de choisir un élément qui serait intimement lié à l'ethos dans ce genre de discours.

La répartition entre les deux candidats montre qu'Hollande est le candidat qui emploie davantage le pronom je. Ceci est peut-être normal puisqu'il est le candidat qui est le moins connu des deux ; il a besoin de montrer qu'il a la capacité d'assumer le rôle de président et que son message est crédible. Les je-dits qui dominent chez les deux sont les je de l'action et de la force, les je de l'idéologue, les je du leadeur humble. Hollande domine fort dans la catégorie je-rassemblement et Sarkozy est celui qui présente le plus de je-autorité. Sarkozy est également le candidat qui emploie le plus le je-négation même si la répartition dans cette catégorie est assez équilibrée entre les deux ; les deux sont conscients de se défendre contre les ethos pré-discursifs qui leur sont attribués mais ils utilisent avant tout le je-négation pour sous-entendre un prédiscursif dévalorisant de l'adversaire en se présentant en même temps comme l'opposé à cette image. Tout pour soigner la crédibilité. Sarkozy est aussi le candidat qui utilise le plus les je-montrés du positionnement du pouvoir, ce qui semble assez naturel vu sa position de président sortant dont la crédibilité repose beaucoup sur son rôle et ses acquis en tant que chef de l'État.

Nous avons analysé comment les je-montrés dénotent le positionnement dans le discours ; ces je auront ainsi le statut d'unités métadiscursives. Il s'agit de formules qui expriment l'acte de maintenir la parole, d'interrompre l'autre, de s'excuser pour interrompre l'autre, de commenter et de renforcer ses propres dits, comme dans « Laissez-moi terminer ", " je vais vous répondre ", etc. Ces $j e$ introduisent des actes de langages au sens propre : les "dires » y sont des "faires ». Ainsi les discours des candidats sont aussi des exercices de pouvoir : l'interaction devient non seulement un champ de bataille des idées mais aussi une démonstration de la force et du pouvoir de la parole. Pour exécuter ce pouvoir, ces métadiscours sont très efficaces.

\subsection{Les je suivis de négation modifient, négocient, adoucissent}

À la présentation de soi à travers les je que font les candidats s'ajoutent ainsi les je combinés avec négation. Les je des énoncés niés présentent indirectement les candidats : en prononçant ce que je ne suis pas, il est sous-entendu soit I) ce que l'autre pense que je suis : c'est un ethos prédiscursif ( $\mathrm{pdv} \mathrm{I})$ que le candidat ( je ») veut réfuter parce qu'il n'est pas d'accord sur cette image; soit 2) ce que je pense que (à la différence 
de moi-même) l'autre candidat est : c'est un ethos pré-discursif défavorisant ( $\mathrm{pdv} \mathrm{I})$ que l'un des candidats attribue à l'autre et duquel il (« je ») se distancie. En d'autres termes, les je-négations permettent au locuteur de se présenter comme une personne crédible. Soit le candidat étant responsable de la réfutation rectifie l'image erronée que l'autre se fait de lui, soit le candidat présente - indirectement - et se distancie à la fois d'une image de l'autre. Dans les deux cas, le candidat nie un ethos pré-discursif, sous-jacent. Mais puisqu'il s'agit de l'ethos, fondé de jugements de valeurs et dépendant de l'auditoire et du contexte, et puisqu'il s'agit ici d'une confrontation entre deux adversaires, la négation négocie - au lieu de réfuter - plutôt cet ethos.

\subsection{Ethos et scènes d'énonciation}

En prenant en considération différents aspects de la scène d'énonciation du débat politique, constatons ainsi que les ethos d'un débat ne sont pas seulement formés par les choix langagiers conscients des candidats (peut-être à l'aide des conseilleurs des médias), mais sont également le résultat d'une négociation à l'intérieur du discours interactif même. Ainsi, les ethos je-dits peuvent être confirmés, parodiés, mis en doute, rectifiés ; si la présentation de soi est mise en question, elle peut être de nouveau réaffirmée. La scène englobante du débat présume également la négociation. Il en est ainsi étant donné que le duel verbal (dont il est question) pour se présenter du bon côté, afin de persuader les spectateurs du bien fondé de son programme politique, est l'élément central de tous les débats politiques et constitue ce qui décide leur fonction textuelle : c'est un discours polémique et argumentatif.

Cette interaction-compétition explique aussi, au niveau de la scène générique, la haute fréquence du pronom je dans les débats présidentiels par rapport aux autres interactions (Fiala \& Leblanche 2004); les jemontrés sont souvent des formules ritualisées qui concernent la maintenance de la parole et l'organisation du discours : " je dis ", " je vais vous dire », " je rappelle », " je vais y venir », " je termine », etc. Les je-dits, par contre, évoquent des thèmes et des engagements présidentiels : « Je veux fermer Fessenheim pour deux raisons ", "Moi, je protège les enfants de la République » (Hollande) et " Je veux mobiliser l'argent public », "j'ai la passion de la France et que je souhaite vous conduire dans ce monde difficile » (Sarkozy). En revenant à la scène englobante et à la visée persuasive, les candidats ne peuvent pas laisser passer les contre-images négatives lancées par l'autre candidat, mais sont censés essayer de les réfuter, ce qui explique le rôle central des « je » et négations dans le débat. 
Pour gagner la bataille, chacun des candidats essaie, au niveau de la scénographie, de distribuer les rôles du discours d'après des schémas de pouvoir, tels que père-fils patron-employé, maitre-élève, autoritéignorant, en se donnant à soi-même soit le rôle du puissant et du savant, soit le rôle du maltraité ou de l'offensé. Les deux rôles peuvent en effet évoquer l'ethos de la crédibilité.

\section{Remarques finales}

Dans cette étude, nous avons catégorisé les je-ethos d'après les verbes accompagnant le pronom de la première question. Constatons cependant que l'analyse quantitative a ses limites quant à un phénomène rhétorico-discursif, puisque l'ethos est aussi l'affaire d'une négociation et d'une interprétation. L'impact rhétorique de la présentation de soi est conçu par ceux qui écoutent et regardent ; la négation par l'un des candidats de l'ethos de l'autre devient une négociation de l'éthos. L'impact rhétorique de la présentation de soi est conçu par ceux qui écoutent et regardent.

Dans nos études antérieures sur la négation en tant que stratégie d'argumentation (Roitman 2013), nous avons étudié comment les réfutations des arguments des autres renforcent l'argumentation des candidats dans l'interaction. Dans les deux cas, nous sommes partie du postulat que la négation est un marqueur de polyphonie qui stratifie les énoncés en deux points de vue ainsi que les enjeux rhétoricoargumentatifs de l'exploitation de cette structure linguistique. Les études se chevauchent mais prennent deux per-spectives différentes, l'une voulant décrire la réfutation comme outil d'argumentation entre deux candidats et l'autre, cette étude-ci, voulant décrire l'impact rhétorique de l'emploi de la négation dans la présentation du JE empirique.

\section{Notes}

I. La présente étude est fondée sur le projet antérieur «Cinq élections présidentielles en France (1974-2007). Les débats de l'entre-deux-tours à la télévision: l'argumentation et contre-argumentation ". Notre matériel se compose des six derniers débats télévisés pour les élections présidentielles, I974-20I2 : Giscard d'Estaing/Mitterrand (I974) ; Mitterrand/Giscard d'Estaing (I98I) ; Mitterrand/Chirac (I988) ; Chirac/Jospin (I995) ; Sarkozy/Royal (2007); Hollande/Sarkozy (20I2) (Éditions Boucher; INA). Les deux derniers débats ont été enregistrés par nos propres moyens. Ils ont été transcrits par les journaux Libération (2007) et Le Monde (20I2) et sont disponibles en ligne. Ces 
transcriptions ont été revues et corrigées par nous-même ; toutefois, certains éléments tels que les termes d'adresse, les marqueurs discursifs, tels que les confirmations, "d'accord ", " bien sûr ", "oui», "non», ainsi que les répliques des deux journalistes, entre autres choses, ont souvent été omis.

2. Véronis est associé à http://politicosphere.blog.lemonde.fr/ qui fait des analyses quantitatives de phénomènes linguistiques du politique sur le Web en collaboration avec Linkfluence, une entreprise de technologie linguistique : http://fr.linkfluence.net/

3. Dans un sens très large, on pourrait étudier l'ethos en tant que phénomène de la sociologie (Fusulier 20II), ou l'ethos "du corps " (Fontanille 2007). Cette conception de l'ethos inclut aussi tout ce qui relève du visuel, de la sémiologie, et du corporel, notamment les gestes et le comportement, etc. Dans la pragmatique américaine de Brown \& Levinson (I987), l'ethos renvoie aux normes d'interaction dans une communauté socio-culturelle : il y aura ainsi un " ethos des Suédois ", par exemple.

4. Aristote traduit par Kennedy (I99I).

5. Pour le même concept, Bourdieu (I982) se sert de la notion " ethos préalable » dans son ouvrage Ce que parler veut dire.

6. Il est intéressant de noter l'évolution de la terminologie dans la recherche d'Amossy (I999, 2000, 2010). En I999 et 2000 elle parle de "l'ethos » et de "l'image de soi » et en 20 Io se sert, en s'inspirant de Goffman (I973), du terme "présentation de soi ». Même si au fond nous sommes assez proche de l'idée classique, à savoir que ce sont les mêmes concepts - bonnes mœurs, statut social - qui attribuent de la crédibilité à une personne, ce changement terminologique témoigne d'une évolution socio-culturelle ; la société est devenue une scène où tout le monde peut se "présenter ", ce qui arrive aussi. La conscience de l'impact qu'on peut obtenir via les chaînes de média est si grande que la présentation de soi est devenue toute une industrie. Les conseillers de communication aident les politiciens à dessiner la personnalité qu'ils souhaitent envoyer au public.

7. Les catégories présentées dans le texte sont des abréviations des catégories du Tableau I.

8. Si un énoncé avec un je-dit est réfuté on aura soit une négation polémique soit une négation descriptive, avec une "présupposition pragmatique " sousjacente (Ducrot I984; Givón 978 : 80). Ce sont les négations des je-dits avec une "présupposition pragmatique » qui nous intéressent quant à l'analyse de l'ethos pré-discursif. Si par contre un énoncé je-montré est nié il y a une négation métalinguistique, dans le sens de Ducrot (I984) et Horn (I989), c'est-àdire une négation qui porte sur les mots mêmes de la parole et non pas sur un argument sous-jacent. La négation métalinguistique rejette les présuppositions, 
elle ne suit pas la loi d'abaissement et inverse l'orientation argumentative normale de la négation sur une échelle descendante. Voir Ducrot (I984) et Horn (1989) pour les tests d'enchaînements et les différentes orientations argumentatives des différents types de négations.

\section{Références}

Adam, Jean-Michel. I999. "Images de soi et schématisation de l'orateur: Pétain et de Gaulle en juin I940 ». In : Ruth Amossy (dir.) Images de soi dans le discours. La construction de l'ethos. Lausanne : Delachaux \& Niestlé. IOI-I 26.

Amossy, Ruth. 1999 (dir.) Images de soi dans le discours. La construction de l'éthos. Lausanne-Paris : Delachaux \& Niestlé.

Amossy, Ruth. 2000. L'Argumentation dans le discours. Discours politique, littérature d'idées, fiction. Paris : Nathan.

Amossy, Ruth. 20I0. La présentation de soi. Ethos et identité verbale. Paris : Presses Universitaires de France.

Barthes, Roland. I970. "L'ancienne rhétorique ». Communications, I6, I970. I72-223. http://www.persee.fr/web/revues/home/prescript/article/ commo588-80I 8 (2013-06-19)

Chudzińska, Yasmine \& Pierre Bourdieu. 1983. « Ce que parler veut dire. L'économie des échanges linguistiques ". Mots, 7 : I 5 5-I6I. http://www.persee.fr/web/revues/home/prescript/article/motso $243-6450$ (2013-06-I9)

Brown, Penelope \& Stephen C. Levinson 1987. Politeness: Some Universals in Language Usage. Studies in interactional sociolinguistics. Cambridge: Cambridge University Press.

Charaudeau, Patrick. 200I. "Visées discursives, genres situationnels et construction textuelle ». In : Analyse des discours. Types et genres, Éd. Universitaires du Sud, Toulouse. Consulté le I9 juin 2013 sur le site de Patrick Charaudeau - Livres, articles, publications. http://www.patrick-charaudeau.com/Visees-discursives-genres, $83 . h t m l$

Charaudeau, Patrick \& Dominique Maingueneau. 2002. Dictionnaire de l'analyse du discours. Paris : Éditions du Seuil.

Ducrot, Oswald. 1984. Le Dire et le dit. Paris : Éditions de Minuit.

Fiala, Pierre \& Jean-Michel Leblanc. 2004. " Autour de JE présidentiel ». Louvain-La-Neuve : JADT 2004. http://lexicometrica.univ-Paris3.fr/jadt/ jadt2004/pdf/JADT_068.pdf (20I3-07-05) 
Fontanille, Jacques. 2007. "Avant-propos : émotion et sémiose » et " Ethos, pathos et persuasion : le corps dans l'argumentation. Le cas du témoignage ». In : Jacques Fontanille (dir.). Les Émotions et configurations dynamiques. Semiotica. Revue de l'Association internationale de sémiotique, I63. Berlin/ New York : Mouton de Gruyter. I-9 et 85-I09.

Fusulier, Bernard. 20 I I. "Le concept d'ethos ». Recherches sociologiques et anthropologiques, 42 (I) : 97-I09. URL : http://rsa.revues.org/66I (20I3-06-I9)

Givón, Talmy. I978 " Negation in Languages: Pragmatics, Function, Ontology » Syntax and Semantics, 9. New York : Academic Press. 69-I I 2.

Goffman, Erving. I973. La mise en scène de la vie quotidienne. La présentation de soi. Paris : Minuit.

Horn, Laurence. I989. A Natural History of Negation. Chicago : University of Chicago Press.

Nølke, Henning, Kjersti Fløttum \& Coco Norén. 2004. ScaPoLine. La théorie scandinave de la polyphonie linguistique. Paris : Kimé.

Leblanc, Jean-Michel \& William Martinez. 2005. "Positionnements énonciatifs dans les vœux présidentiels sous la cinquième République », Corpus 4. http://corpus.revues.org/index347.html (2013-07-05)

Kennedy, George, A. I991. (Aristotle). On rhetoric. A Theory of Civic discourse. Newly translated, with Introduction, Notes and Appendices. New York/Oxford : Oxford University Press.

Kerbrat-Orecchioni, Catherine. I980. L'énonciation. Paris : Armand Colin.

Kerbrat-Orecchioni, Catherine. 2005. Le discours en interaction. Paris : Armand Colin.

Maingueneau, Dominique. 2002. "L'ethos, de la rhétorique à l'analyse du disours ». <http://dominique.maingueneau.pagesperso-orange.fr/intro_company.html> (2013-06-I9)

Maingueneau, Dominique. I999. " Ethos, scénographie, incorporation ». In : Ruth Amossy (dir.) Images de soi dans le discours, La construction de l'ethos. Lausanne : Delachaux \& Niestlé. 75-Ior.

Maingueneau, Dominique. I998. Analyser les textes de communication. Paris : Dunod.

Marchand, Pascal \& Pierre-Olivier Dupuy. 2009. " Débat de l'entre-deux tours 2007 : la conquête de l'espace lexical ». Mots : les langages du politique. 89 (2007 : débats pour l'Elysée). I05-I I7.

Marchand, Pascal \& Pierre-Olivier Dupuy. 20I . "Confrontation et positionnement dans les duels de l'entre-deux tours : une approche lexicométrique ». 
In : Marcel Burger, Jérôme Jacquin \& Raphaël Micheli (dir.). La parole politique en confrontation dans les médias. Bruxelles : De Boeck. I29-I47.

Marchand, Pascal \& Pierre-Olivier Dupuy. 20I2. "Le débat d'entre les deux tours (2 mai 20I2)». http://pascalmarchand.fr/ spip.php?article 55 (2013-06-19)

Roitman, Malin \& Françoise Sullet-Nylander. 20ıo. « Voix de campagne présidentielle : quelques observations sur la question et la réfutation dans le débat télévisé Royal-Sarkozy (2 mai 2007) ». In : Abecassis Michael \& Ledegen, Gudrun (dir.) Les voix des français : à travers l'histoire, l'école et la presse. Oxford : Peter Lang.

Roitman, Malin. 20I3. "Construire son argumentation sur la réfutation du discours de l'autre : les débats de l'entre-deux-tours ». In : Sullet-Nylander, Françoise, Gunnel Engwall \& Hugues Engel (dir.). La linguistique dans tous les sens. Stockholm : Kungl. Vitterhets Historie och Antikvitets Akademien.

Véronis, Jean. 2007. « Moi, François Hollande ». http://blog.veronis. fr/20I 2/05/debat-moi-francois-hollande.html (2013-06-I9)

Véronis, Jean. 2007. Les mots de Nicolas Sarkozy. Paris : Éditions du Seuil).

Véronis, Jean. 20I2. "Je revient ". http://politicosphere.blog.lemonde. $\mathrm{fr} / 20 \mathrm{I}_{2} / \mathrm{O}_{3} / 23$ / sarkozy-je-revient/ (2OI3-I9) 